\title{
Metabolomics Characterizes the Effects and Mechanisms of Quercetin in the Nonalcoholic Fatty Liver Disease Development
}

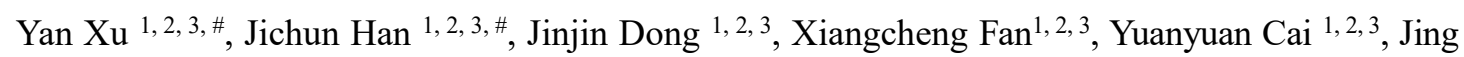
$\mathrm{Li}^{4}$, Tao Wang ${ }^{5,6}$, Jia Zhou ${ }^{1,2,3, *}$, Jing Shang ${ }^{1,2,3, *}$

${ }^{1}$ School of Traditional Chinese Pharmacy, China Pharmaceutical University, Nanjing 211198, China

${ }^{2}$ State Key Laboratory of Natural Medicines, China Pharmaceutical University, Nanjing 210009, China

${ }^{3}$ Jiangsu Key Laboratory of TCM Evaluation and Translational Research, China Pharmaceutical University, Nanjing 211198, China

${ }^{4}$ School of Life Science and Technology, China Pharmaceutical University, Nanjing 210009 , China.

${ }^{5}$ Jiangsu Key Laboratory of Drug Screening, China Pharmaceutical University, Nanjing 211198, China

6 Jiangsu Center for Pharmacodynamics Research and Evaluation, China Pharmaceutical University, Nanjing 210009, China

${ }^{\#}$ These authors contributed equally to this work.

*Correspondence should be addressed to Jing Shang (shangiing21cn@cpu.edu.cn) and Jia Zhou (cpuzj_2016@163.com).

\begin{abstract}
As metabolomics is widely used in the study of disease mechanisms, more and more studies have found that metabolites play an important role in the occurrence of
\end{abstract}


diseases. The aim of this study is to investigate the effects and mechanisms of quercetin in high-fat-sucrose diet (HFD)-induced nonalcoholic fatty liver disease (NAFLD) development using nontargeted metabolomics. A rat model of NAFLD was established by feeding with a HFD for 30 and 50 days. Results indicated quercetin exhibited hepatoprotective activity in HFD-induced NAFLD rats in 30 days by regulating fatty acids related metabolites (adrenic acid, etc.), inflammation related metabolites (arachidonic acid, etc.), oxidative stress related metabolites (2-hydroxybutyric acid) and other differential metabolites (citric acid, etc.). However, quercetin couldn't improve NAFLD in 50 days maybe it couldn't reverse the inflammation condition induced by long-term high-fat diet. These data indicate that dietary quercetin may be beneficial to NAFLD at early stages. Furthermore, combining metabolomics and experimental approaches opens up avenues of effects and mechanisms of drugs for complex diseases.

Keywords: nonalcoholic fatty liver disease; high fat-sucrose diet; metabolomics; HPLC-QTOF-MS; quercetin

\section{Introduction}

Nonalcoholic fatty liver disease (NAFLD) is acknowledged to be the hepatic manifestation of obesity and metabolic syndrome, which includes a wide spectrum of liver diseases, ranging from simple fatty liver to nonalcoholic steatohepatitis (NASH), and these may eventually progress to liver cirrhosis, and hepatocellular carcinoma [1]. NAFLD presents an increasing incidence worldwide, which is a serious threat to people's health [2]. The pathogenesis of NAFLD involves many mechanisms, such as lipid peroxidation, inflammatory factor damage, oxidative stress and insulin resistance $[3,4]$. Lipid accumulation and inflammation have been reported to contribute to the development of NAFLD $[5,6]$. Several studies have also discovered that some compounds can reduce NAFLD by anti-inflammation and inhibition of lipid accumulation, such as alpinetin and rutin [7,8]. Therefore, finding a drug that can 
reduce inflammation and inhibit lipid accumulation may be a logical therapeutic strategy for the treatment of NAFLD.

Recent studies indicate that some small metabolites are assossiated with NAFLD. Researches showed that some fatty acids such as palmitic acid [9], docosahexaenoic acid and eicosapentaenoic acid [10], changed in NAFLD, could promote lipid accumulation. Arachidonic acid could mediate inflammation which involved in the development and progression of NAFLD [11]. In HFD-induced NAFLD, changed succinic acid and citric acid level may cause disturbances in the mitochondrial TCA cycle, thereby promoting increased oxidative stress, impairment of hepatic mitochondrial function, and the release of cytokines, eventually leading to hepatitis [12]. Alterations in levels of bile acids showed the positive correlation to NAFLD in both rodents and humans [13]. Metabolomics is defined as the quantitative measurement of the time-related small metabolites of multicellular systems to pathophysiological stimuli or a genetic modification [14]. At present, metabolomics technology has been widely used in the study of NAFLD drugs, such as pharmacodynamic evaluation, drug screening and mechanism research [15]. Therefore, it is a logical therapeutic strategy for finding and studying NAFLD drugs by using metabolomics techniques.

Quercetin is one of the most abundant dietary flavonoids that presents a wide variety of biological function [16]. Beneficial effects of quercetin have been reported on lipid accumulation, inflammation, fibrosis, nitrosative/oxidative stress and insulin resistance associated to NAFLD [17]. And studies have found that quercetin can effectively alleviate NAFLD injury [18]. However, the altered metabolites of quercetin on NAFLD is poorly understood. Therefore, our study aimed to explore the effects and mechanisms of quercetin on NAFLD metabolites by metabolomics technology.

\section{Results}




\subsection{Effects of Quercetin on liver injury in HFD-induced NAFLD development}

To investigate the effects of quercetin on HFD-induced NAFLD injury, we first determined the serum AST and ALT levels in control, model and quercetin groups in 30 and 50 days. Liver injury was confirmed by the HE staining which demonstrated the HFD-induced hepatic vacuoles, lipid droplets and hepatocyte swelling (Fig. 1A) in M30 and M50 compared to corresponding control group and M50 was more serious than M30. Significant increases of serum AST and ALT levels $(p<0.01)$ were found in M30 and M50 compared to corresponding control group and both levels in M50 were higher than M30 (Figure 1B-C). Treatment with quercetin significantly decreased AST and ALT levels in serum $(p<0.05)$, and also significantly inhibited hepatic vacuoles, lipid droplets and hepatocyte swelling compared to corresponding model group in 30 and 50 days. Also Q30 had better effects on HFD-induced NAFLD injury than Q50. Taken together, these data suggest that quercetin has better protective effects on hepatic injury in HFD-induced NAFLD rats in 30 days than 50 days.

2.2. Metabolomic Profile of serum extracts in the control, model and quercetin groups from different time points

To understand the reason why quercetin has better protective effects on hepatic injury in HFD-induced NAFLD rats in 30 days than 50 days. We used the HPLC-QTOF based untargeted metabolomics to study. In this study, we detected 641 positive-mode features and 263 negtive-mode features after peak alignment and removal of missing values. The ions with variable importance in the projection (VIP) values $>1.0$ and $p<0.05$ were considered the potential differential metabolites. 277 postive-mode ions and 132 negtive-mode ions had significantly changed $(p<0.05$, VIP $>1$ ). Principal component analysis (PCA) was initially used as an unsupervised statistical method to study the metabolomic differences between control, model and quercetin groups from different time points. PCA score plots (Fig. 2A-B) showed a clear separation between the control and model groups from different points, 
indicating that the high-fat-sucrose diet could lead to significant variations in the serum metabolic profiling and model group was more apart from control group in 30 days than 50 days. In addition, the position of quercetin in 30 days was more neareast to control group, indicating quercetin in 30 days can best reverse the changed metabolic patterns caused by HFD than 50 days (Fig. 2C-D).

The differential metabolites were identified by searching in METLIN and HMDB database, and then searched the related metabolic pathway in KEGG database. The differential metabolites contributing to the separation of the model group from the corresponding control group and reversed differential metabolites in treatment with quercetin in 30 and 50 days, along with their significance values (VIP value, and $p$ value), are summarized in Table S1-2.

2.3. Characterization of Differential Metabolite Patterns Associated with HFD-induced NAFLD development

We identified 19 and 17 metabolites that were significantly changed in M30 and M50 compared to correponding control group respectively. Metabolites significantly changed in model group compared to corresponding control group could be roughly divided into the following categories: (1) fatty acids related, (2) inflammation related, (3) oxidative stress related, and (4) other categories. Then PCA sorce plots analyst on each catagory were showed in Figure 3A-H. There was a better separation on fatty acid related metabolites between control and model group in 30 days than 50 days while separation on inflammation and oxidative stress related metabolites between control and model group in 50 days were better than 30 days. PCA score plots on other metabolites showed these metabolites could separate control and model group in both 30 days and 50 days equally.

As shown in the heat map (Fig. 3I), fatty acid related metabolites including adrenic acid, docosahexaenoic acid, palmitic acid, linoleic acid, oleic acid and eicosapentaenoic acid were significantly downregulated by HFD and these 
metabolites decreased more obvious in M30 than M50. Oxidative stress and inflamation related metabolites including $\mathrm{p}$-cresol sulfate, indoxyl sulfate, 12(S)-HPETE and 12-HETE were significantly upregulated while 2-hydroxybutyric acid and arachidonic acid were significantly downregulated by HFD. P-cresol sulfate, indoxyl sulfate, 12(S)-HPETE and 12-HETE increased more obvious in M50 than M30 while 2-hydroxybutyric acid and arachidonic acid decreased less obvious in M50 than M30. Other increased differential metabolites in model group compared to corresponding control group were chenodeoxycholic acid glycine conjugate, taurocholic acid, glycocholic acid, succinic acid, 15(S)-hydroxyeicosatrienoic acid, alpha-dimorphecolic acid and 9,10,13-TriHOME. In contrast, citric acid and L-tyrosine decreased in model group compared to corresponding control group. Taken together, metabolites changed in HFD-induced NAFLD in 30 days mainly involved in fatty acids while 50 days mainly involved in inflammation.

2.4. Metabolic consequences of quercetin treatment in rats with HFD-induced NAFLD development

The PCA sorce plots analyst on fatty acids, inflammation, oxidative stress and other catagory in 30 and 50 days were showed in Figure 4A and Figure C. Results showed that Q30 could better reverse the changed pattern caused by HFD whether from the fatty acids, inflammation, oxidative stress and other aspects. To further evaluate the reversed effect of the potential metabolites by administration of quercetin more intuitively, we detected changes in 13 potential metabolites. These data suggest that quercetin may reverse 5 fatty acids (adrenic acid, docosahexaenoic acid, palmitic acid, oleic acid and eicosapentaenoic acid), 2 inflammation related metabolites (12(S)-HPETE and arachidonic acid), 1 oxidative stress related metabolites (2-hydroxybutyric acid) and other 5 metabolites (15(S)-hydroxyeicosatrienoic acid, alpha-dimorphecolic acid, 9,10,13-TriHOME, citric acid and chenodeoxycholic acid glycine conjugate) in 30 days (Figure 4B). In contrast, quercetin could only reverse palmitic acid, 2-hydroxybutyric acid and 12(S)-HPETE in 50 days (Figure 4D). 
Taken together, quercetin could reverse fatty acids related metabolites in 30 days while the effects on reversing inflammation related metabolites were not so obvious in 50 days.

2.5. Observation of effects of Quercetin on hepatic steatosis and inflammation in HFD-induced NAFLD development

To observe the effects of quercetin on lipid accumulation and inflammation in HFD-induced NAFLD in 30 and 50 days, we determined the serum lipid levels, liver lipid levels and liver inflammation in control, model and quercetin groups in 30 and 50 days. As shown in Figure 5A-D, compared with corresponding control group, the levels of TG, TC and LDL in serum were significantly increased $(p<0.01)$, while the HDL level in serum was significantly decreased in model group $(p<0.01)$ and the degree on the changes in 30 were higher than 50 days. Compared with corresponding model group, treatment with quercetin decreased the levels of TG, TC and LDL in serum $(p<0.05)$, and increased the HDL level $(p<0.05)$ better in 30 than 50 days. And liver lipid levels measured by Oil Red O-staining (Figure 5E) were consistent with serum lipid levels by ELISA. The positive kupffer cells were observed by CD68 staining to determine the inflammation status. As shown in Figure 5F, the liver that underwent HFD showed a significant increase in the number of CD68 positive marking kupffer cells in 50 days while not so obvious in 30 days,and treatment with quercetin couldn't effectively inhibit this phenotype in M50. Taken together, these data suggest that quercetin administration could alleviate lipid accumulation in HFD-induced NAFLD. However, it couldn't effectively alleviate inflammation in HFD-induced NAFLD.

\section{Discussion}

The high fat-sucrose diet model is a widely used murine model of NAFLD [19]. 
HFD produce the phenotype like steatosis and inflammation within 4 weeks in SD rats, and these conditions continue to progres [20]. These indicate that the HFD is a good model to study NAFLD and the mechanism of action of potential therapeutic agents. In the present study, the NAFLD model in rats was successfully reproduced by feeding HFD for 30 and 50 days, and we found that HFD induced more serious liver injury in 50 days than 30 days according to serum ALT, AST and HE staining. Consistent with some research results [21], we found quercetin could ameliorate liver injury symptom caused by NAFLD through decreased serum ALT, AST and alleviated hepatic vacuoles, swelling. Interestingly, Q30 had a better effects on liver injury than Q50. Taken together, these results indicate that quercetin was able to improve liver injury in NAFLD better in 30 days than 50 days.

Metabolomics, systematic analysis of all metabolites and metabolomic pathway in a given biological system, has been increasingly recognized in biomarker discovery and understanding disease mechanism. As an important indicator of peripheral circulation that can reflect the real-time changes in various life activities accurately, serum has become a hot research topic in metabolomics studies [22]. To understand the reason why quercetin has better protective effects on hepatic injury in HFD-induced NAFLD rats in 30 days than 50 days, we use metabolomics technology to study this phenomenon and potential mechanisms. HFD led to significant variations in serum metabolic profiling in rats, which indicated metabolic disorders in the model group rats. And PCA from 30 days had a better separation between control and model group than 50 days. 19 and 17 metabolites were found to significantly change between the model and control groups in 30 and 50 days respectively which were consistent with PCA results. Serum metabolic profiling results showed that quercetin can better reverse the abnormal metabolic patterns caused by HFD in 30 days than 50 days. From the reversed metabolites, we can also see Q30 could reverse 13 metabolites and Q50 only 3 metabolites.

The relative content of differential metabolites associated with NAFLD and quercetin treatment were examined and then compared. The networks correlated with 
the potential metabolites, the main disturbed metabolic pathway related to NAFLD and the possible metabolic mechanisms of quercetin treatment are summarized in Figure 6. Here, we will discuss the reliable and abundant evidence for the potential differential metabolites associated with NAFLD and quercetin treatment.

This study revealed that the levels of adrenic acid, linoleic acid, docosahexaenoic acid, eicosapentaenoic acid, palmitic acid and oleic acid decreased in HFD-induced NAFLD development. The significant depletion of fatty acids indicate a reduction in fatty acid oxidation and triglyceride release from the liver with a consequent increase in triglyceride synthesis that may contribute significantly to the development of triglyceride accumulation in hepatocytes [23]. These changes are similar to those described by metabolomic analysis in NAFLD [24]. These fatty acids decreased more significantly in 30 days than 50 days which indicated lipid accumulation in M30 was more serious than M50. Quercetin can better improve NAFLD in 30 days than 50 days maybe due to its better effects on reversing fatty acids related metabolites such as adrenic acid, docosahexaenoic acid, eicosapentaenoic acid, palmitic acid and oleic acid. Simultaneously, Oil Red O-staining as well as serum TG, TC, LDL and HDL levels confirmed the more serious lipid accumulation and better effects of quercetin in 30 days than 50 days.

Moreover, we noticed that 12(S)-HPETE and 12(S)HETE increased while arachidonic acid decreased in HFD-induced NAFLD development. Arachidonic acid is a polyunsaturated fatty acid which can mediate inflammation and is a key player in the synthetic pathway for pro-inflammatory series 2 prostaglandins and leukotrienes [25]. 12-HPETE is produced by the non-enzymatic oxidation of arachidonic acid through the 12-lipoxygenase pathway and metabolize to produce 12-HETE. They both participate in host defense reactions and pathophysological conditions such as immediate inflammation [26]. Research have also shown that one biochemical pathway that is likely to be relevant but has not yet been extensively studied in NAFLD, is the eicosanoid generating lipoxygenases pathway 12-LOX [27]. The lower level of arachidonic acid in model group probably reflects the body adaption to 
inflammation. These inflammation related metabolites changed more significantly in 50 days than 30 days which indicated inflammation in M50 was more serious than M30. Quercetin can better improve NAFLD in 30 days than 50 days maybe due to its better effects on reversing inflammation related metabolites such as arachidonic acid and 12(S)-HPETE, while inflammation in M50 maybe too serious to be reversed by Q50. Simultaneously, CD68 positive marking kupffer cells confirmed the more serious inflammation status in NFFLD in 50 days and better effects of quercetin in 30 days than 50 days.

We also discovered that $\mathrm{p}$-Cresol sulfate and Indoxyl sulfate increased while 2-Hydroxybutyric acid decreased in HFD-induced NAFLD development. Protein-bound uremic toxins, $\mathrm{p}$-cresol sulfate and indoxyl sulfate, increase oxidative stress and adversely affect chronic kidney disease progression and cardiovascular complications [28]. 2-Hydroxybutyric acid has been shown as an early marker for both insulin resistance and impaired glucose regulation. The underlying biochemical mechanisms may involve increased lipid oxidation and oxidative stress [29]. More recently it has been noted that elevated levels of 2-hydroxybutyric acid in the plasma is a good marker for early-stage type II diabetes [30]. Compared to corresponding control group, the lower level of 2-hydroxybutyric acid in model group represented an adaptive response to lipid oxidation or oxidative stress. These oxidative stress related metabolites changed more significantly in 50 days than 30 days which indicated oxidative stress in M50 was more serious than M30. Quercetin can better improve NAFLD in 30 days than 50 days maybe due to its better effects on reversing oxidative stress related metabolites such as 2-hydroxybutyric acid, while oxidative stress in M50 maybe too serious to be reversed by Q50.

In the liver, cholesterol participates in primary bile acid biosynthesis to form primary conjugated bile acids such as glycocholate, taurocholate, glycochenodeoxycholate, and taurochenodeoxycholate [31]. Metabolomic analysis has revealed significantly increased serum levels of glycocholate, taurocholate, glycochenodeoxycholate in patients with NASH compared with healthy patients [32]. 
In HFD-induced NAFLD, the increased hepatic cholesterol may raise up bile acids levels due to cholestasis, then increased bile acids can lead to an activition of lipogenic genes and develop hepatic steatosis [33].

Alpha-dimorphecolic acid is an endogenous fatty acid peroxisomal proliferator-activated receptor-gamma agonist synthesized from linoleic acid. It can activate PPAR-gamma in human endothelial cells or adipocytes and increase plasminogen activator inhibitor type-1 expression correlating with the risk for myocardial infarction and venous thrombosis. A study showed that alpha-dimorphecolic acid was significantly increased in NASH patients with steatosis [34]. 15(S)-Hydroxyeicosatrienoic acid (15S-HETrE) is the in vivo metabolite of gamma-linolenic acid, and it has been reported to suppress cyclooxygenase-2 over expression and/or prostaglandin E2 biosynthesis [35], inhibits [(3)H]thymidine uptake in parallel with the upregulation of peroxisome proliferator-activated receptor-gamma expression to exert antiproliferative activities [36]. 9,10,13-TriHOME was another metabolite formed as lipoxygenase-catalyzed products of linoleic acid. In our study, higher levels of alpha-dimorphecolic acid, 15S-HETrE and 9,10,13-TriHOME indicated lipid metabolism dysfunction in HFD-induced NAFLD.

TCA impairments have been correlated with multiple diseases where oxidative stress plays a key role. Lipid accumulation-induced mitochondria DNA damage correlates with mitochondrial dysfunction and increased oxidative stress in skeletal muscle and liver, which are associated with the induction of endoplasmic reticulum stress markers ER stress, protein degradation and apoptosis [37]. Additionally, an ineffective TCA cycle might be an adaptive response directed at diminishing reactive oxygen species production and extend cellular longevity [38]. Inhibition of the citrate transporter, which regulates the plasma citrate flux into the liver, was also reported to increase plasma citrate, increase hepatic mitochondrial biogenesis, and prevent diet-induced hepatic steatosis and hepatic insulin resistance [39]. Plasma tyrosine levels were positively associated with the severity of steatosis in the liver [40], the origins and mechanisms of tyrosine metabolism dysregulation in hepatic steatosis 
remain poorly elucidated. In present study, succinic aicd increased while citric acid and L-Tyrosine decreased in HFD-induced NAFLD indicated that TCA cycle and tyrosine metabolism were affected by HFD feeding. Quercetin could better improve NAFLD in 30 days than 50 days maybe due to its better effects on reversing other metabolites such as chenodeoxycholic acid glycine conjugate, citric acid, 15(S)-hydroxyeicosatrienoic acid, alpha-dimorphecolic acid and 9,10,13-TriHOME.

In summary, quercetin exhibited hepatoprotective activity in HFD-induced NAFLD rats in 30 days by regulating fatty acids related metabolites (adrenic acid, docosahexaenoic acid, eicosapentaenoic acid, palmitic acid and oleic acid), inflammation related metabolites (arachidonic acid and 12(S)-HPETE), oxidative stress related metabolites (2-hydroxybutyric acid) and other differential metabolites (chenodeoxycholic acid glycine conjugate, citric acid, 15(S)-hydroxyeicosatrienoic acid, alpha-dimorphecolic acid and 9,10,13-TriHOME). However, quercetin couldn't improve NAFLD in 50 days maybe it couldn't reverse the inflammation condition induced by long-term high-fat diet. Furthermore, combining metabolomics and experimental approaches opens up avenues of effects and mechanisms of drugs for complex diseases.

\section{Materials and Methods}

\subsection{Animal Model}

Experiments were performed with six-weeks-old male Sprague-Dawley (SD) rats weighing 180 220 g (Shanghai Super-B\&K laboratory animal Corp. Ltd., Shanghai, China) under controlled environmental conditions (temperature of $23 \pm 2{ }^{\circ} \mathrm{C}$, relative humidity of $50-70 \%$, and $12 \mathrm{~h}$ light/dark cycle) with ad libitum access to food and water. This study was approved by the Science and Technology Department of Jiangsu Province (SYXK(SU)2016-0011) and all animal experiments complied with the standard ethical guidelines under the ethical committees mentioned above. After adaptation for one week, all rats were randomly divided into six groups $(n=6)$ as 
follows: (a) Control group: fed with a basal diet $(360 \mathrm{kcal} / 100 \mathrm{~g}$; fat, $13.3 \mathrm{~g} / 100 \mathrm{~g}$; protein, $26.2 \mathrm{~g} / 100 \mathrm{~g}$; carbohydrate, $60.5 \mathrm{~g} / 100 \mathrm{~g}$ ); (b) Model group: fed with a high fat-sucrose diet (HFD) (506.8 kcal/100 g; lard, $10 \mathrm{~g} / 100 \mathrm{~g}$; cholesterol, $2 \mathrm{~g} / 100 \mathrm{~g}$; egg yolk power, $5 \mathrm{~g} / 100 \mathrm{~g}$; sucrose, $10 \mathrm{~g} / 100 \mathrm{~g}$; propylthiouracil, $2 \mathrm{~g} / 100 \mathrm{~g}$; basal diet, $72.8 \mathrm{~g} / 100 \mathrm{~g})$. (c) Quercetin treatment group: fed with HFD and given quercetin $(50 \mathrm{mg} / \mathrm{kg}$ per day). The quercetin was suspended in a $0.5 \%$ carboxymethylcellulose solution and was administrated by oral gavage once per day from the twentyth day . Rats in the control group and model group were given the $0.5 \%$ carboxymethylcellulose solution only. The basal diet and high fat-sucrose diet were provided by the Jiangsu Xietong Medical and Biological Corporation (Nanjing, China). Food intake and body weight were recorded daily during the experiment period. Six rats from each group were fasted overnight and sacrificed under anesthesia on 30 and 50 days for collection of blood and liver.

\subsection{Serological Analyses}

Whole blood were immediately collected and then allowed to clot. After centrifugation at $1200 \mathrm{~g}$ for $15 \mathrm{~min}$ at $4^{\circ} \mathrm{C}$, the resultant serum samples were separated and stored individually at $-80^{\circ} \mathrm{C}$ for subsequent analyses. Aspartate and alanine transaminases (AST and ALT) were measured to assess the hepatic injury. Total triglycerides (TG), total cholesterol (TC), low-density lipoprotein (LDL), and high-density lipoprotein (HDL) were evaluated the lipid changes. All above-mentioned indexes were measured by commercially available kits (Nanjing Jiancheng Bioengineering Institute, Nanjing, China).

\subsection{Liver Histopathological Examination}

Liver pathological analysis and semi-quantitative evaluation was carried out. Briefly, liver tissues were fixed in $4 \%$ paraformaldehyde for 4 hours and dehydrated in 
a series of ethanol and embedded in paraffin wax. Sections (4-mm-thick) were stained with hematoxylin-eosin (HE) staining before being analyzed under an Olympus biological microscope (BX53, Tokyo, Japan). Oil Red O-stained lipid droplets of the fresh liver samples were analyzed to quantify lipid content by cell imaging under an Olympus-BX53 biological microscope. Areas of stained droplets were determined using Image $\mathbf{J}$ software and normalized to the areas of rat hepatocytes. CD68 (1:800, ab31630, abcam, Cambridge, UK) were used for histological analysis under a light microscope Olympus-BX53.

\subsection{Serum Sample Preparation}

Serum sample $(100 \mu \mathrm{L})$ was mixed with $300 \mu \mathrm{L}$ cold $\mathrm{MeOH}$ and then vortexed for $3 \mathrm{~min}$ to precipitate the protein. After centrifugation $\left(13300 \mathrm{rpm}\right.$ at $4{ }^{\circ} \mathrm{C}$ for 15 $\min$ ), $300 \mu 1$ of supernatant was transferred to another vial and then dried at room temperature using a Thermo SPD1010 SpeedVac Kit (Thermo, USA). The dried residue was dissolved in $100 \mu \mathrm{L}$ water/acetonitrile (4:1) solution and ultrasonicated for $15 \mathrm{~min}$ to dissolve the sample. After centrifugation (13300 rpm at $4{ }^{\circ} \mathrm{C}$ for $15 \mathrm{~min}$ ), the supernatant was transferred into the autosampler vial and a $2 \mu \mathrm{L}$ aliquot was injected for LC-MS analysis. For quality control, equal amounts of each sample aliquots were pooled and applied to monitor the stability of analytical performance in the platform.

\subsection{LC-MS Analysis}

Serum metabolic profiling was performed using a 1260 Rapid Resolution Liquid Chromatography (RRLC) system coupled to a 6530 quadrupole time of flight (Q-TOF) MS (Agilent, Santa Clara, CA). Chromatographic separations for processed serum was achieved on a XSelect HSS T3 column $(2.1 \times 100 \mathrm{~mm}, 2.5 \mu \mathrm{m}$, Waters $)$ maintained at $40{ }^{\circ} \mathrm{C}$ and a pump flow rate of $0.4 \mathrm{~mL} / \mathrm{min}$. The mobile phase system was 
composed of A (water with $0.1 \%$ formic acid) and B (acetonitrile). The gradient elution program was as follows: $0-2 \mathrm{~min}, 2 \% \mathrm{~B} ; 2-7 \mathrm{~min}, 2-60 \% \mathrm{~B} ; 7-20 \mathrm{~min}$, $60-100 \%$ B ,20-23 min, 100\% B.

The electrospray ionization (ESI) source was set in positive and negative mode with the data being collected between $50-1000 \mathrm{~m} / \mathrm{z}$ in centroid mode using the high-resolution mode $(4 \mathrm{GHz})$. For the positive ionization mode, the MS parameters were as follows: the fragmentor at $130 \mathrm{~V}$, nebulization gas at $40 \mathrm{psi}$, nozzle voltage at $0 \mathrm{~V}$, the vcap at $4000 \mathrm{~V}$, drying gas flow rate at $8 \mathrm{~L} / \mathrm{min}$, and temperature at $325^{\circ} \mathrm{C}$, sheath gas flow rate at $12 \mathrm{~L} / \mathrm{min}$, and temperature at $350{ }^{\circ} \mathrm{C}$. For the negative ionization mode, the parameters were the same except the nozzle voltage set to $500 \mathrm{~V}$. Reference masses at $\mathrm{m} / \mathrm{z} 121.050873,922.009798$ in positive mode and 112.985587, 1033.988109 in negative mode were introduced for accurate mass calibration.

\subsection{Data Processing and Analysis}

The acquired mass spectrometry data(.d) were exported to data format (.mzdata) files by MassHunter Workstation Software (Version B.06.00, Agilent Technologies). Using R Foundation for Statistical Computing, data pretreatment procedures such as nonlinear retention time alignment, peak discrimination, filtering, alignment, and matching were performed in XCMS package (http://metlin.scripps.edu/download/). The ion features that are present in less than $80 \%$ samples were screened out. Open database sources, including the KEGG, MetaboAnalyst, Human Metabolome Database, and METLIN, were used to identify metabolic pathways.

\subsection{Statistical Analysis}

Results are expressed as the mean \pm SEM for all pathophysiological data by Student's t-test using GraphPad Prism software (UK), with $p$ values less than 0.05 regarded as significant. The metabolomic variables responsible for the discrimination 
were identified by the principal components analysis (PCA) and statistical differences between the compared groups were assessed by the Mann-Whitney U test. A $p$ value $<0.05$ and VIP $>1$ was considered statistically significant. PCA analysis and heat map were all conducted using MetaboAnalyst.

Author Contributions: Conceptualization, Xiangcheng Fan; Data curation, Yuanyuan Cai; Formal analysis, Yan Xu and Jichun Han; Investigation, Tao Wang; Methodology, Yan Xu and Jia Zhou; Project administration, Jing Shang; Software, Jinjin Dong and Jing Li; Writing - original draft, Yan Xu and Jichun Han; Writing review \& editing, Jing Shang.

Funding: This work was supported by the Applied Basic Research Programs of Qinghai Province (No. 318 Y229461211), Science and Technology Plan Projects in Qinghai Province (No.2015-ZJ-733), A prospective joint research project of Jiangsu Province (BY2016-078-02).

Conflicts of Interest: The authors declare that they have no conflict of interest.
Abbreviations
PCA principal components analysis
HFD high-fat-sucrose diet
NAFLD nonalcoholic fatty liver disease
NASH nonalcoholic steatohepatitis

\section{References}


1. Rinella, M.E. Nonalcoholic fatty liver disease: a systematic review. JAMA. 2015, 313, 2263-273. doi: 10.1001/jama.2015.5370.

2. Sweet, P.; Khoo, T.; Nguyen, S. Nonalcoholic Fatty Liver Disease. Prim Care. 2017, 44, 599-607. doi: 10.1016/j.pop.2017.07.003.

3. Xu, Y.; Yang, C.; Zhang, S.; Li, J.; Xiao, Q.; Huang, W. Ginsenoside Rg1 protects against non-alcoholic fatty liver disease by ameliorating lipid peroxidation, endoplasmic reticulum stress, and inflammasome activation. Biol Pharm Bull. 2018, 41, 1638-1644. doi: 10.1248/bpb.b18-00132.

4. Theocharidou, E.; Papademetriou, M.; Reklou, A.; Sachinidis, A.; Boutari, C.; Giouleme, O. The role of PCSK9 in the pathogenesis of non-alcoholic fatty liver disease and the effect of PCSK9 inhibitors. Curr Pharm Des. 2018. doi: $10.2174 / 1381612824666181010123127$.

5. Zaki, S.M.; Abdel, Fattah, S.; Hassan, D.S. The differential effects of high-fat and high fructose diets on the liver of male albino rat and the proposed underlying mechanisms. Folia Morphol (Warsz). 2018. doi: 10.5603/FM.a2018.0063.

6. Pan, Z.G.; An, X.S. SARM1 deletion restrains NAFLD induced by high fat diet (HFD) through reducing inflammation, oxidative stress and lipid accumulation. Biochem Biophys Res Commun. 2018, 498, 416-423. doi: 10.1016/j.bbrc.2018.02.115.

7. Zhou, Y.; Ding, Y.L.; Zhang, J.L.; Zhang, P.; Wang, J.Q.; Li, Z.H. Alpinetin improved high fat diet-induced non-alcoholic fatty liver disease (NAFLD) through improving oxidative stress, inflammatory response and lipid metabolism. Biomed Pharmacother. 2018, 97, 1397-1408. doi: 10.1016/j.biopha.2017.10.035.

8. Panchal, S.K.; Poudyal, H.; Arumugam, T.V.; Brown, L. Rutin attenuates metabolic changes, nonalcoholic steatohepatitis, and cardiovascular remodeling in high-carbohydrate, high-fat diet-fed rats. $J$ Nutr. 2011, 141, 1062-1069. doi: 10.3945/jn.111.137877.. 
9. Cao, H.; Gerhold, K.; Mayers, J.R.; Wiest, M.M.; Watkins, S.M.; Hotamisligil, G.S. Identification of a lipokine, a lipid hormone linking adipose tissue to systemic metabolism. Cell. 2008, 134, 933-944. doi: 10.1016/j.cell.2008.07.048.

10. Shang, T.; Liu, L.; Zhou, J.; Zhang, M.; Hu, Q.; Fang, M.; Wu, Y.; Yao, P.; Gong, Z. Protective effects of various ratios of DHA/EPA supplementation on high-fat diet-induced liver damage in mice. Lipids Health Dis. 2017, 16, 65. doi: 10.1186/s12944-017-0461-2.

11. Ma, K.; Chen, Y.; Liang, X.; Miao, J.; Zhao, Q. Inhibition of 5-lipoxygenase inhibitor zileuton in high-fat diet-induced nonalcoholic fatty liver disease progression model. Iran J Basic Med Sci. 2017, 20, 1207-1212. doi: 10.22038/IJBMS.2017.9482.

12. Begriche, K.; Massart, J.; Robin, M.A.; Bonnet, F.; Fromenty, B. Mitochondrial adaptations and dysfunctions in nonalcoholic fatty liver disease. Hepatology. 2013, 58, 1497-1507. doi: 10.1002/hep.26226.

13. Lake, A.D.; Novak, P.; Shipkova, P.; Aranibar, N.; Robertson, D.; Reily, M.D.; Lu, Z.; Lehman-McKeeman, L.D.; Cherrington, N.J. Decreased hepatotoxic bile acid composition and altered synthesis in progressive human nonalcoholic fatty liver disease. Toxicol Appl Pharmacol. 2013, 268, 132-140. doi: 10.1016/j.taap.2013.01.022.

14. Bujak, R.; Struck-Lewicka, W.; Markuszewski, M.J.; Kaliszan, R. Metabolomics for laboratory diagnostics. J Pharm Biomed Anal. 2015, 113, 108-120. doi: 10.1016/j.jpba.2014.12.017.

15. Safaei, A.; Arefi Oskouie, A.; Mohebbi SR.; Rezaei-Tavirani, M.; Mahboubi, M.; Peyvandi, M.; Okhovatian, F.; Zamanian-Azodi, M. Metabolomic analysis of human cirrhosis, hepatocellular carcinoma, non-alcoholic fatty liver disease and non-alcoholic steatohepatitis diseases. Gastroenterol Hepatol Bed Bench. 2016, 9, $158-173$. 
16. Rauf, A.; Imran, M.; Khan, I.A.; Ur-Rehman, M.; Gilani, S.A.; Mehmood, Z.; Mubarak, M.S. Anticancer potential of quercetin: A comprehensive review. Phytother Res. 2018, 32, 2109-2130. doi: 10.1002/ptr.6155.

17. Wang, J.; Miao, M.; Zhang, Y.; Liu, R.; Li, X.; Cui, Y.; Qu, L. Quercetin ameliorates liver injury induced with Tripterygium glycosides by reducing oxidative stress and inflammation. Can J Physiol Pharmacol. 2015, 93, 427-433. doi: 10.1139/cjpp-2015-0038.

18. Davoodi, I.; Rahimi, R.; Abdollahi, M.; Farzaei, F.; Farzaei, M.H.; Memariani, Z.; Najafi, F. Promising effect of Rosa damascena extract on high-fat diet-induced nonalcoholic fatty liver. J Tradit Complement Med. 2017, 7, 508-514. doi: 10.1016/j.jtcme.2017.01.008.

19. Goedeke, L.; Bates, J.; Vatner, D.F.; Perry, R.J.; Wang, T.; Ramirez, R.; Li, L.; Ellis, M.W.; Zhang, D.; Wong, K.E.; et al. Acetyl-CoA Carboxylase Inhibition Reverses NAFLD and Hepatic Insulin Resistance but Promotes Hypertriglyceridemia in Rodents. Hepatology. 2018, 68, 2197-2211. doi: 10.1002/hep.30097.

20. Young, K.; Aguilar, M.; Gish, R.; Younossi, Z.; Saab, S.; Bhuket, T.; Liu, B.; Ahmed, A.; Wong, R.J. Lower rates of receiving model for end-stage liver disease exception and longer time to transplant among nonalcoholic steatohepatitis hepatocellular carcinoma. Liver Transpl. 2016, 22, 1356-1366. doi: 10.1002/1t.24507.

21. Pisonero-Vaquero, S.; Martínez-Ferreras, Á.; García-Mediavilla, M.V.; Martínez-Flórez, S.; Fernández, A.; Benet, M.; Olcoz, J.L.; Jover, R.; González-Gallego, J.; Sánchez-Campo,s S. Quercetin ameliorates dysregulation of lipid metabolism genes via the PI3K/AKT pathway in a diet-induced mouse model of nonalcoholic fatty liver disease. Mol Nutr Food Res. 2015, 59, 879-893. doi: 10.1002/mnfr.201400913. 
22. Liu, L.; Aa, J.; Wang, G.; Yan, B.; Zhang, Y.; Wang, X.; Zhao, C.; Cao, B.; Shi, J.; Li, M.; et al. Differences in metabolite profile between blood plasma and serum. Anal Biochem. 2010, 406, 105-112. doi: 10.1016/j.ab.2010.07.015.

23. Videla, L.A.; Rodrigo, R.; Araya, J.; Poniachik, J. Oxidative stress and depletion of hepatic long-chain polyunsaturated fatty acids may contribute to nonalcoholic fatty liver disease. Free Radic Biol Med. 2004, 37, 1499-1507.

24. Sham, T.T.; Zhang, H.; Mok, D.K.W.; Chan, S.W.; Wu, J.; Tang, S.; Chan, C.O. Chemical Analysis of Astragali Complanati Semen and Its Hypocholesterolemic Effect Using Serum Metabolomics Based on Gas Chromatography-Mass Spectrometry. Antioxidants (Basel). 2017, 6, pii: E57. doi: 10.3390/antiox6030057.

25. Levin, G.; Duffin, K.L.; Obukowicz, M.G.; Hummer,t S.L.; Fujiwara, H.; Needleman, P.; Raz A. Differential metabolism of dihomo-gamma-linolenic acid and arachidonic acid by cyclo-oxygenase-1 and cyclo-oxygenase-2: implications for cellular synthesis of prostaglandin E1 and prostaglandin E2. Biochem J. 2002, $365,489-496$.

26. Powell, W.S.; Rokach, J. Biosynthesis, biological effects, and receptors of hydroxyeicosatetraenoic acids (HETEs) and oxoeicosatetraenoic acids (oxo-ETEs) derived from arachidonic acid. Biochim Biophys Acta. 2015, 1851, 340-355. doi: 10.1016/j.bbalip.2014.10.008.

27. Samala, N.; Tersey, S.A.; Chalasani, N.; Anderson, R.M.; Mirmira, R.G. Molecular mechanisms of nonalcoholic fatty liver disease: Potential role for 12-lipoxygenase. $J$ Diabetes Complications. 2017, 31, 1630-1637. doi: 10.1016/j.jdiacomp.2017.07.014.

28. Sun, C.Y.; Cheng, M.L.; Pan, H.C.; Lee, J.H.; Lee, C.C. Protein-bound uremic toxins impaired mitochondrial dynamics and functions. Oncotarget. 2017, 8, 77722-77733. doi: 10.18632/oncotarget.20773. 
29. Gall, W.E.; Beebe, K.; Lawton, K.A.; Adam, K.P.; Mitchell, M.W.; Nakhle, P.J.; Ryals, J.A.; Milburn, M.V.; Nannipieri, M.; Camastra, S.; et al. alpha-hydroxybutyrate is an early biomarker of insulin resistance and glucose intolerance in a nondiabetic population. PLoS One. 2010, 5, e10883. doi: 10.1371/journal.pone.0010883.

30. Li, X.; Xu, Z.; Lu, X.; Yang, X.; Yin, P.; Kong, H.; Yu, Y.; Xu, G. Comprehensive two-dimensional gas chromatography/time-of-flight mass spectrometry for metabonomics: Biomarker discovery for diabetes mellitus. Anal Chim Acta. 2009, 633, 257-262. doi: 10.1016/j.aca.2008.11.058.

31. Chow, M.D.; Lee, Y.H.; Guo, G.L. The role of bile acids in nonalcoholic fatty liver disease and nonalcoholic steatohepatitis. Mol Aspects Med. 2017, 56, 34-44. doi: 10.1016/j.mam.2017.04.004.

32. Kalhan, S.C.; Guo, L.; Edmison, J.; Dasarathy, S.; McCullough, A.J.; Hanson, R.W.; Milburn, M. Plasma metabolomic profile in nonalcoholic fatty liver disease. Metabolism. 2011, 60, 404-413. doi: 10.1016/j.metabol.2010.03.006.

33. Martin, I.V.; Schmitt, J.; Minkenberg, A.; Mertens, J.C.; Stieger, B.; Mullhaupt, B.; Geier, A. Bile acid retention and activation of endogenous hepatic farnesoid-X-receptor in the pathogenesis of fatty liver disease in ob/ob-mice. Biol Chem. 2010, 391, 1441-1449. doi: 10.1515/BC.2010.141.

34. Feldstein, A.E.; Lopez, R.; Tamimi, T.A.; Yerian, L.; Chung, Y.M.; Berk, M.; Zhang, R.; McIntyre, T.M.; Hazen, S.L. Mass spectrometric profiling of oxidized lipid products in human nonalcoholic fatty liver disease and nonalcoholic steatohepatitis. J Lipid Res. 2010, 51, 3046-3054. doi: 10.1194/jlr.M007096.

35. Pham, H.; Banerjee, T.; Ziboh, V.A. Suppression of cyclooxygenase-2 overexpression by $15 \mathrm{~S}$-hydroxyeicosatrienoic acid in androgen-dependent prostatic adenocarcinoma cells. Int J Cancer. 2004, 111, 192-197.

36. Pham, H.; Banerjee, T.; Nalbandian, G.M.; Ziboh, V.A. Activation of peroxisome 
proliferator-activated receptor (PPAR)-gamma by 15S-hydroxyeicosatrienoic acid parallels growth suppression of androgen-dependent prostatic adenocarcinoma cells. Cancer Lett. 2003, 189, 17-25.

37. Yuzefovych, L.V.; Musiyenko, S.I.; Wilson, G.L.; Rachek, L.I. Mitochondrial DNA damage and dysfunction, and oxidative stress are associated with endoplasmic reticulum stress, protein degradation and apoptosis in high fat diet-induced insulin resistance mice. PLoS One. 2013, 8, :e54059. doi: 10.1371/journal.pone.0054059.

38. Calderón-Santiago, M.; Priego-Capote, F.; Galache-Osuna, J.G.; Luque de Castro, M.D. Method based on GC-MS to study the influence of tricarboxylic acid cycle metabolites on cardiovascular risk factors. J Pharm Biomed Anal. 2013, 74, 178-185. doi: 10.1016/j.jpba.2012.10.029.

39. Li, Z.; Erion, D.M.; Maurer, T.S. Model-Based Assessment of Plasma Citrate Flux Into the Liver: Implications for $\mathrm{NaCT}$ as a Therapeutic Target. CPT Pharmacometrics Syst Pharmacol. 2016, 5, 132-139. doi: 10.1002/psp4.12062.

40. Jin, R.; Banton, S.; Tran, V.T.; Konomi, J.V.; Li, S.; Jones, D.P.; Vos, M.B. Amino Acid Metabolism is Altered in Adolescents with Nonalcoholic Fatty Liver Disease-An Untargeted, High Resolution Metabolomics Study. J Pediatr. 2016, 172, 14-19. doi: 10.1016/j.jpeds.2016.01.026. 
$\mathbf{A}$

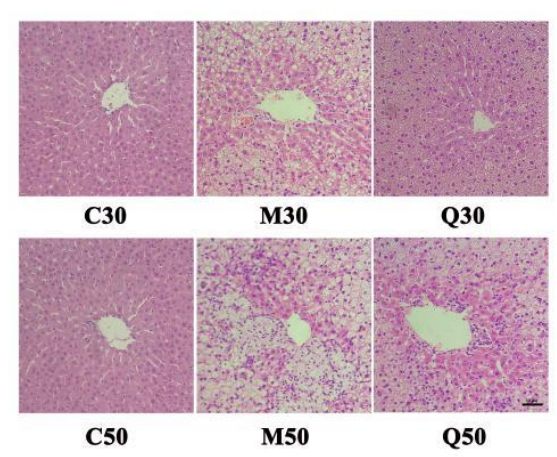

B

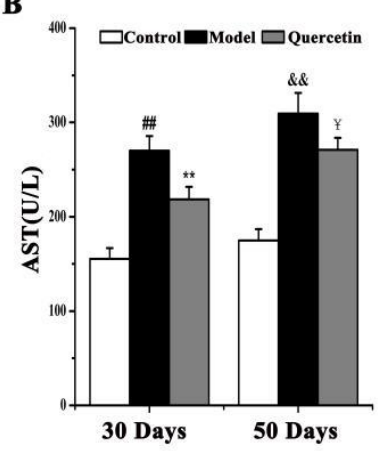

C

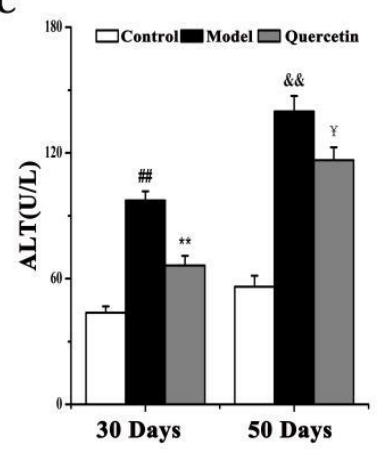

Figure 1. Effects of HFD and quercetin treatment on hepatic injury in NAFLD development. (A) Typical hematoxylin-eosin $(\mathrm{HE})$ staining $(\times 200)$. White and black arrows display fat vacuole of hepatocytes and infiltration of inflammatory cells, respectively; (B) Serum AST and ALT levels. Comparison was made by Student's t-test, ${ }^{\#} p<0.01,{ }^{\&} \& p<0.01$ vs. corresponding control group, ${ }^{* *} p<0.01,{ }^{\$}<0.01,{ }^{\$}<0.05$ vs. corresponding model group. AST, aspartate aminotransferase; ALT, alanine aminotransferase.
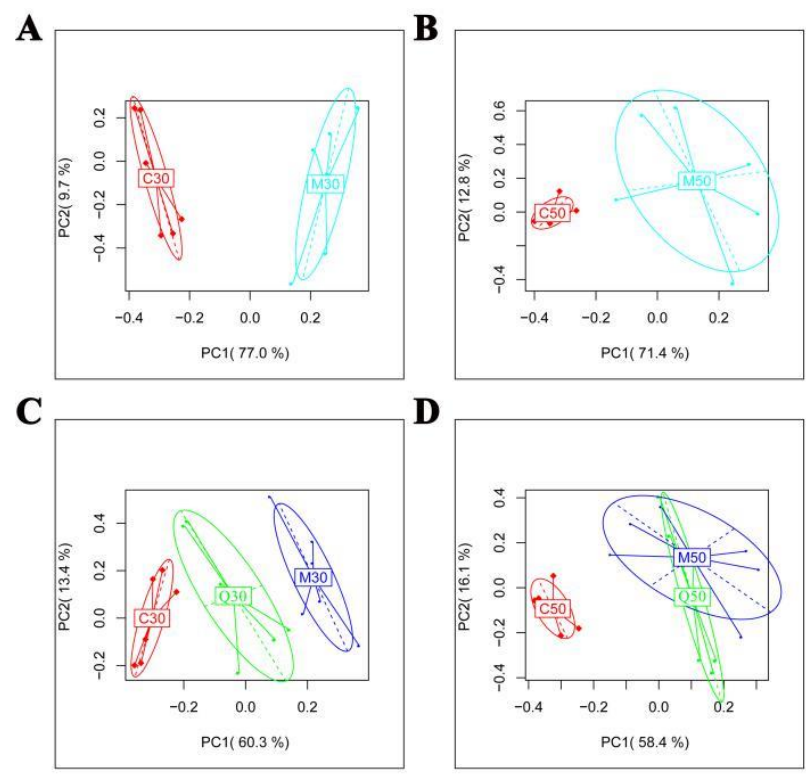

Figure 2. Metabolic profiling of serum samples in control, model and quercetin groups. PCA score plots from control and model groups after 30 days (A) and 50 days (B) feeding; PCA score plots from control, model and quercetin groups after 30 days (C) and 50 days (D) feeding. 

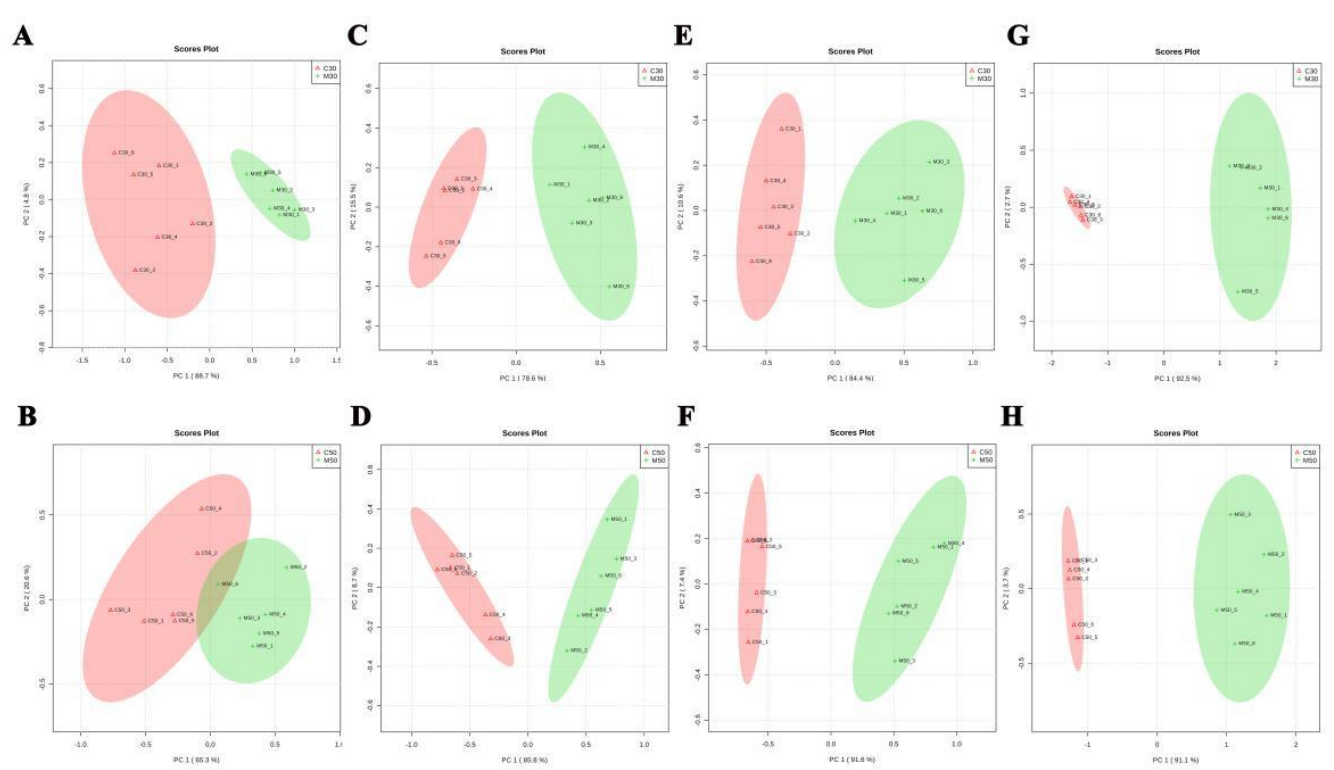

$\mathbf{H}$

Fatty acid

Oxidative Stress

Inflammation
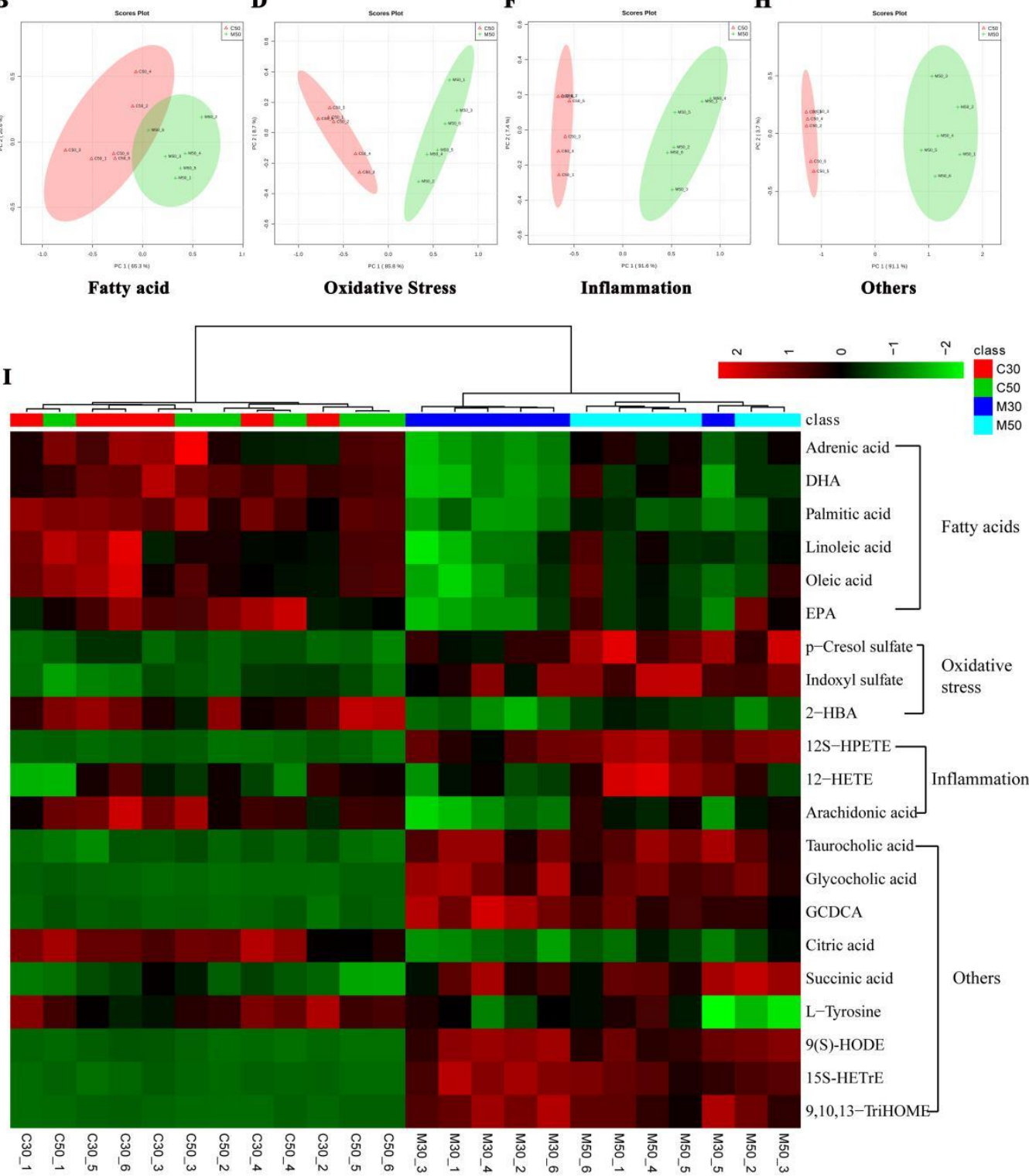

Figure 3. PCA score plots (A-H) and heat map (I) on differential metabolites in HFD-induced NAFLD development. PCA analyst based on classification in fatty acids (A, B), oxidative stress $(C, D)$, inflammation (E, F) and other metabolites $(\mathrm{G}, \mathrm{H})$. The colors in the heat map changing from green to red indicate more metabolites. DHA: docosahexaenoic acid; EPA: eicosapentaenoic acid; 2-HBA: 2-hydroxybutyric acid; GCDCA: chenodeoxycholic acid glycine conjugate; 9(S)-HODE: alpha-dimorphecolic acid; 15(S)-HETrE: 15(S)-hydroxyeicosatrienoic acid. 

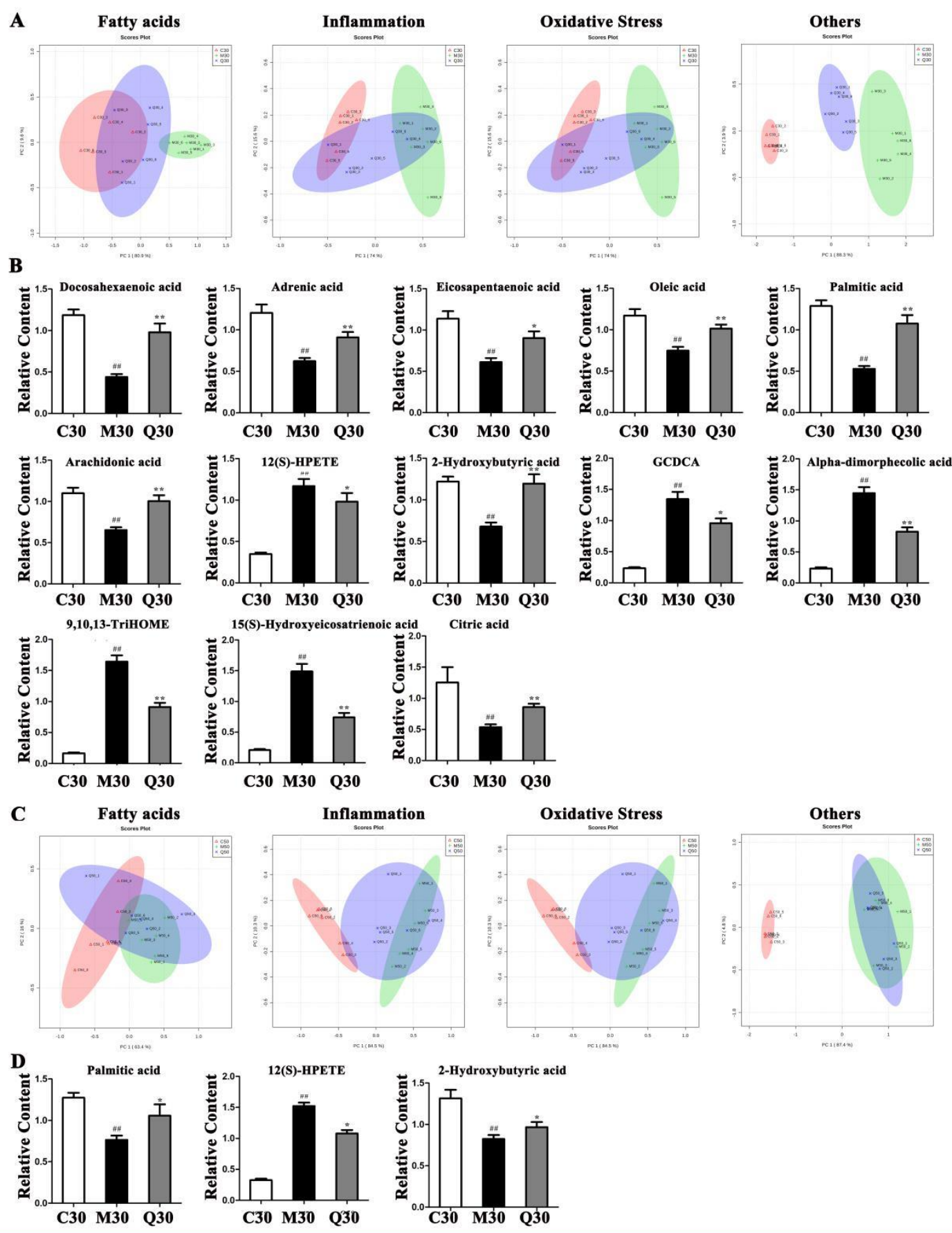

Figure 4. PCA score plots analyst based on different classification in 30 days (A) and 50 days (C). The reversed differential metabolites by quercetin in 30 days (B) and 50 days (D). ${ }^{\#} p<0.01$ vs. corresponding control group. ${ }^{*} p<0.05 ;{ }^{* *} p<0.01$ vs. corresponding model group. 

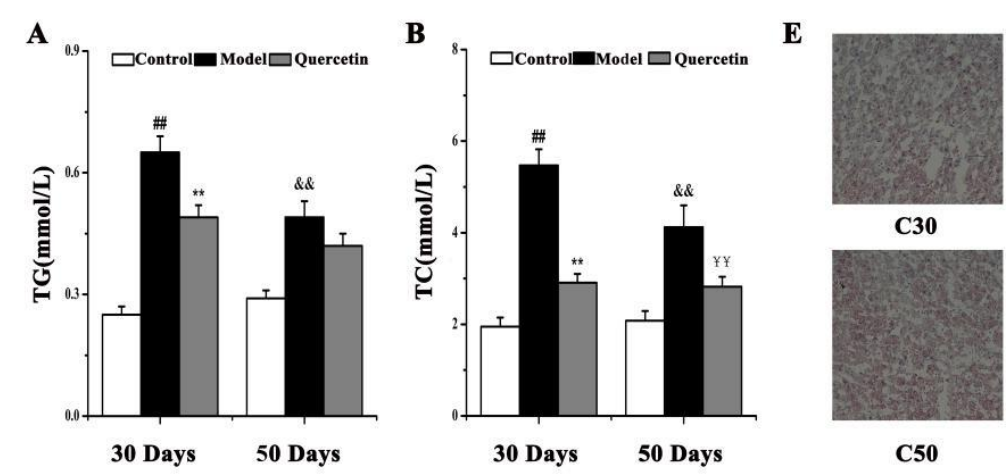

C30

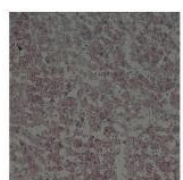

C50

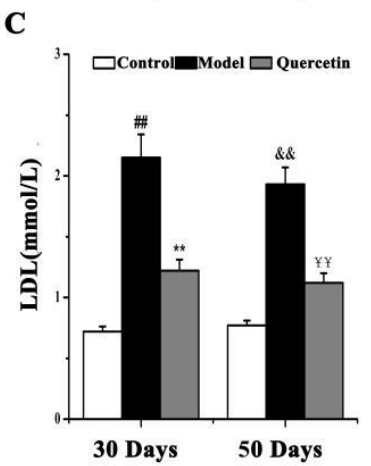

D

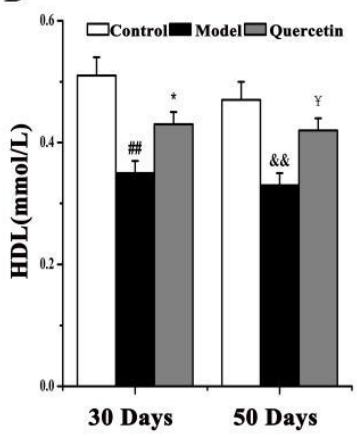

$\mathbf{F}$

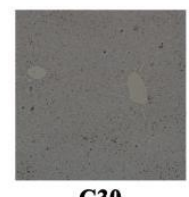

C30

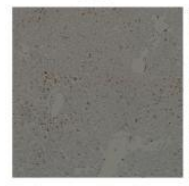

C50

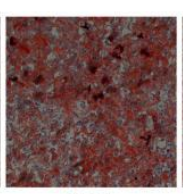

M30

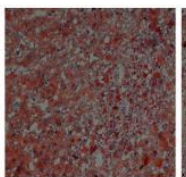

M50

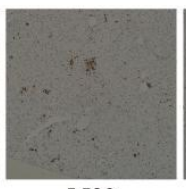

M30

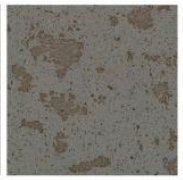

M50

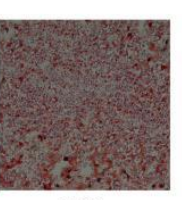

Q30

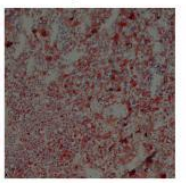

Q50

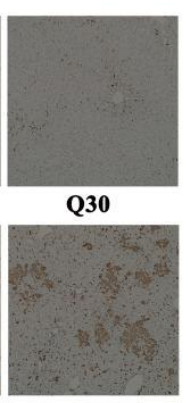

Q50

Figure 5. Lipid accumulation and inflammation status in control, model and quercetin groups in 30 and 50 days. (A) Serum TG, TC, LDL, HDL levels; (B) Oil red O staining $(\times 200)$. Small red circles indicate the formation of large cytoplasmic lipid droplets; (C) CD68 positive kupffer cell ( $\times 100)$. Comparison was made by Student's t-test, ${ }^{\#} p<0.01,{ }^{\& \&} p<0.01$ vs. corresponding control group, ${ }^{* *} p<0.01,{ }^{*} p<0.05{ }^{\$}<0.01,{ }^{\$}<0.05$ vs. corresponding model group. TG, total triglyceride; TC, total cholesterol; HDL, high-density lipoprotein; LDL, low-density lipoprotein. 


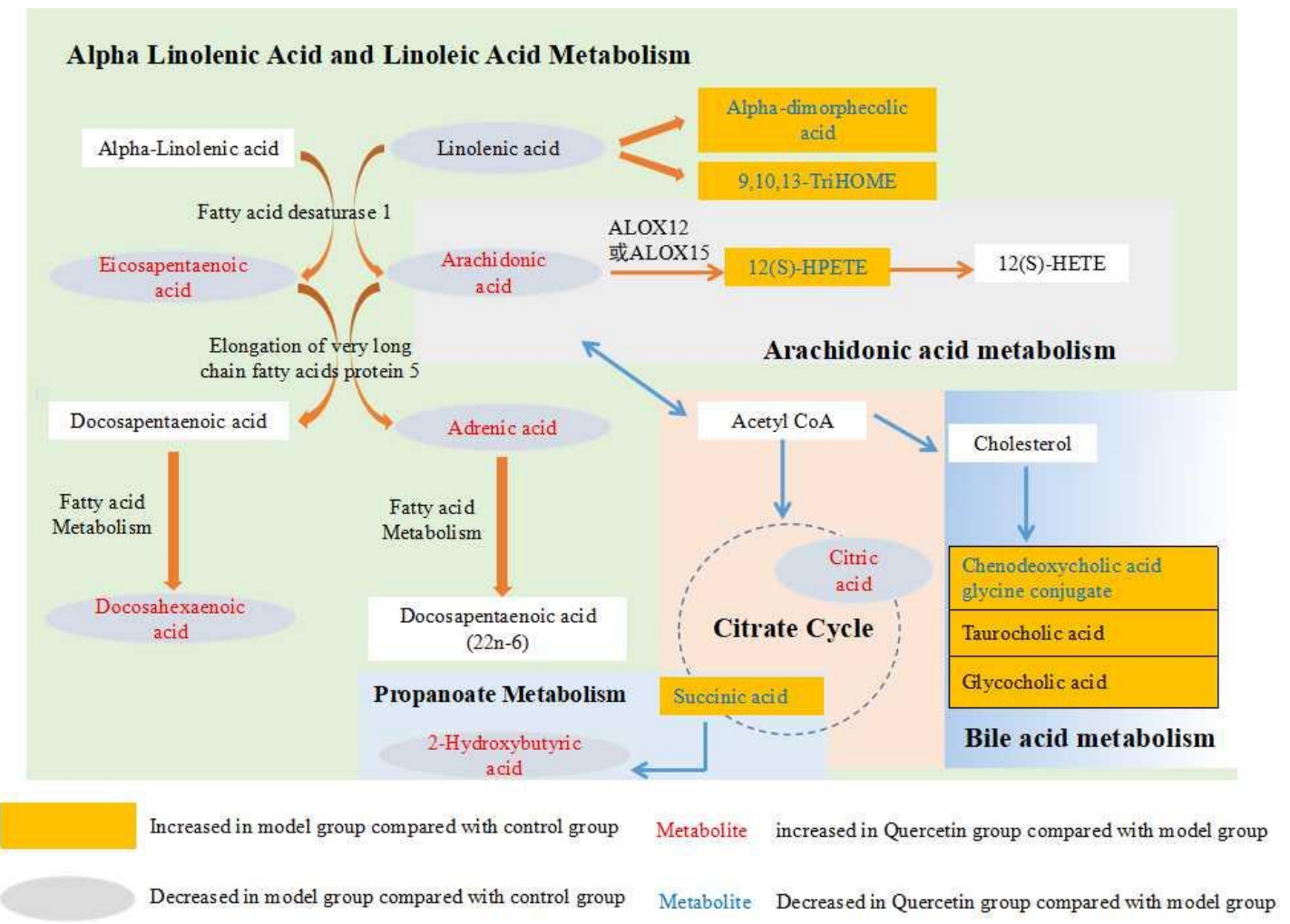

Figure 6. Summary scheme of quercetin-targeted pathways in rats with NAFLD in 30 days. Quercetin administration could ameliorate the fatty liver through regulating the expression of metabolites related to fatty acid and inflammation. Furthermore, the linoleic acid and arachidonic acid related pathways could be regulated by quercetin. The red and blue metabolites represent significant $(p<0.05$, VIP $>1)$ changes induced by quercetin. The red metabolites indicate quercetin-induced upregulation and the blue metabolites indicate downregulation. 